\title{
The support of multidimensional approaches in integrate monitoring for SEA: a case of study
}

\author{
C. M. Torre and M. Selicato \\ Dipartimento di Scienze dell'Ingegneria Civile e dell'Architettura, Politecnico di Bari, Bari, Italy \\ Correspondence to: C. M. Torre (cartorre@yahoo.com) \\ Received: 21 September 2012 - Published in Earth Syst. Dynam. Discuss.: 9 October 2012 \\ Revised: 24 November 2012 - Accepted: 26 November 2012 - Published: 28 January 2013
}

\begin{abstract}
During the centuries, the seaside has represented a crucial pole for future human development and civilization. The use of the sea for transport and trade and the overwhelming availability of food derived from coastal waters have encouraged and strengthened the growth of urban settlements. In the same time, the human pressure menaces to destroy coastal habitats and consequently their carrying capacity that allows for many essential functions.

Low-impact activities are often replaced, on the surface, by new intensive ones that are attractive in the short term, but that in the long term undermine by reducing the resilience of the coast. It is clear that, in a perspective of sustainable development, economically efficient and socially equitable use of coastal areas need to be supported inside strategies to correct these weaknesses. The definition of such strategies and their implementation in the Strategic Environmental Assessment (SEA) is an essential tool for supporting decisions and of monitoring.

The issues of monitoring, more in particular, have been the subject of study and modelling by the use of Dynamic Spatial Data Analysis (DSDA), in the case of the SEA of the Coastal Plan of the Italian Apulia Region, as an information instrument for regulating the anthropogenic changes; a possibility to implement the analysis of environmental sensitivity and propensity to Coastal erosion has been explored, in order to control the level of human pressure on land. The monitoring system should provide an automatic "alert" when the dimension and the velocity of the change of land use overpass some threshold of environmental pressure.
\end{abstract}

\section{Introduction}

It is uneasy to stem the diffusion of inappropriate uses of coastal areas and, indeed, the growing number of users (residents and visitors). In this chapter we analyse the role of Strategic Environmental Assessment (SEA) as support of planning procedure.

SEA is configured as a systematic process for evaluating the environmental consequences of plans and programmes: it permeates the plan/programme and represents a support for management and monitoring. Many authors (Sadler and Verheem, 1996; Partidário, 2000; Sheate, 2010) recognised the need to follow a sequence for implementing the SEA procedure; Fischer (2007), in particular, define what it is, find as many scholars to treat it, what must be done or how it is done in practice.

The Directive 2001/42/EC has been in Europe, the starting pulse, to focus on stages of the SEA, as it gives a way to follow them, explicitly providing monitoring of significant environmental effects of implementing plans and programmes and the possibility of mitigation measures in the application, but it is considered appropriate to "broaden the picture", not limited to environmental monitoring, explaining that it stems from it and what is required for the SEA to have efficacy and is required when evaluation whose results are to be integrated in the post decision, yet also "limit" monitoring key indicators and environmental issues deemed most critical and sensitive that is a step for follow up (Bencardino, 2006).

Although the signs of the Directive on monitoring are limited and have limited the indications from the European guidelines, this applies even more if we refer to the Italian Legislative Framework, that is the reference point for our 
case study: it is necessary to establish guidelines and criteria for monitoring so that the same is effective and VAS with it.

Partidario and Arts (2005) argue that implementing Strategic Environmental Assessment cannot be limited to what is prescribed, or what should be done in the manner as SEA Environmental Report describes and, relevantly, how it should be accompanied by environmental monitoring carried out by means of appropriate indicators.

These aspects are still unclear, especially the transition from theory to practice, because there is still a theoretical debate about definitions, key concepts, approaches, tools, methods and techniques.

Arts (1998) indicates the post-decision phases with the term follow up and provide a definition, as regards both Environmental Impact Assessment (EIA) and SEA, as an ex-post monitoring and evaluating impacts of a project/plan, in order to manage and communicate the environmental performance of the same project/plan.

The monitoring, evaluation of compliance, management and reporting of impacts are also elements of the follow-up according to Marshall (2005).

In a direct follow-up monitoring can be defined as the answer to "what happens after" stages of approval. Practice in planning, indeed, is not as simple as thinking about what might happen at the project level, how it may be easier and easier to administer; size, timing and predictable are well defined, while in the field of strategic decisions are very difficult to see the foreshadowing that is considered decisions based on the intentions or actions planned but provided longterm, you do not have much in reference to what will happen, what will be the embodiment and implementation, if there is a change in current policy and new policies, if implemented will be a project or programme, and what will be your address (Kornov and Thissen, 2000; Cerreta et al., 2012). This is not completely true when we speak about the strategic project and the issues are anyway relevant on a regional scale (e.g., Nuclear Power Station).

As underlined by Morrison-Saunders et al. (2007), a strategic policy can go in whatever direction; not necessarily in a linear trend and not with the same amplitude, we add that the representation of planning as linear or cyclic is a reductionist approach to reality.

Partidario and Arts (2005) suggest that the follow up can be seen as an ex-post evaluation of the consequences of the actions and can have four different dimensions to investigate: in addition to compliance, we consider performance, uncertainty and dissemination. The relationship between such four dimensions and information management during the final phase of monitoring, indicates that you can follow five paths to implement this later step of the SEA:

a. monitoring the changes;

b. assess achievement of stated objectives;

c. evaluate the performance of the initiatives; d. test the compliance of the decision-making process with the provision of the plan and of the SEA;

e. indentifying and assessing the real impacts on the environment and the sustainability strategic initiative.

These five activities lead the dynamic management of a wellperforming Strategic Environmental Assessment.

Each approach is differently profiled from the others and has different objectives and techniques. You can approach by using them individually and mixing them in different phases, depending on the context and on the purpose.

Regarding the steps after making the Directive, they explicitly provide only monitoring and do not provide information on evaluation activities, management and communication, with regard to the impacts component part of the followup, but they are implicit and connected to the first.

The correct approach to the SEA, according to the author, should be carried out on the basis of two main actions: describing the effects but, then, relating them to objectives of sustainability; at each stage of the planning process the two evaluations have a specific function and must be made.

As regards to how to carry out monitoring, the starting idea is that monitoring of significant environmental effects is the only requirement, but if you want to link the plan with the environmental effects it is necessary to know terms and timing of implementation; this means that monitoring must also cover indicators of plan (Selicato et al., 2012).

In reference to the construction and operation of the monitoring system, these are considered important indications of McCallun (1985):

- plan in advance the necessary activities: what needs to be done, by whom and how, stakeholders and coordinate activities;

- be clear about what you are doing;

- manage information so that they are produced and made available;

- provide adequate resources;

- maintain the credibility of those involved in the process.

And Partidario and Arts (2005) reminds us that focus should be:

- first, on the strategic nature of the initiative and its impacts on the direction, timing, scale and consequences of the initiative, the tangibility and concreteness and measurability, and so on;

- second, objectives, implementation and controlling changes, learning, informing and communicating;

- third, on significant issues and approaches necessary: whatever the approach, the monitoring should follow the key indicators, identify areas sensitive to changes 
due to strategic initiative, but first of all be aware of the information available. To implement an effective monitoring system and adhering to the contents and meanings of the European Directive is necessary to verify the existence of a number of conditions. For instance, you must consider which peculiar methodological and contextual elements have to be for the Italian planning system, that is based on rigid procedures and prescriptive rules, with authority whose duties and responsibilities are subdivided among distinct institutional actors: evaluators/planners, policy makers, inspectors, that primarily provided a judgement of compatibility of plans with environmental issues.

As regards monitoring, it should be accompanied by a clear and at the same time flexible planning procedure, finalised to change the issue related to monitored parameters where this has been evidenced as the need to do: but Fisher and Gazzola (2006), at the end, considered that in Italy it is very dangerous to give flexibility to the system, even if it could be useful.

\section{Case study: the monitoring system in the SEA of Apulia Regional Coastal Plan}

Coastal areas can be defined as the connecting line among land and sea, representing a source not only for ecotypes and natural habitat in the environmental perspective, but also for social and economic development.

Maritime cities and natural seaside resources play a strategic role by potentially meeting the needs and wishes of European citizens. In this chapter, we tell about an experiment of monitoring human pressures on costal habitats and settlements by the support of a Dynamic Spatial Data Analysis (DSDA). The occasion is due to the development of the SEA report of the Coastal Plan of the Italian Apulia Region (RCP); the report traces the guidelines for devoting information to check and regulate the anthropogenic changes.

Besides being the extreme eastern region of Italy, Apulia, accounts for about $800 \mathrm{~km}$ of coastline, one of the greater regional coastal developments in Italy (see Fig. 1). The coast is characterised as rocky, in Gargano Peninsula, rock and calcareous in the middle south Adriatic, and finally sandy beaches such as along the Gulf of Taranto in the Ionic Sea. The $98 \%$ of Apulia's coast are bathing. Therefore, the attention to the coast for tourism and recreation is high, and the conflict between activitie development and environmental protection need to be managed by the Regulations of RCP.

The initial idea started by observing the relationship of human pressures and environmental sensitivity and propensity to Coastal erosion. Inside the RCP, the coastal line is subdivided in 28 stripes, called Physiographic Sub-Ambits (PSA), which appear homogeneous according to physiographic aspects and erosion dynamics. Each PSA in the most general case can belong to different municipality.

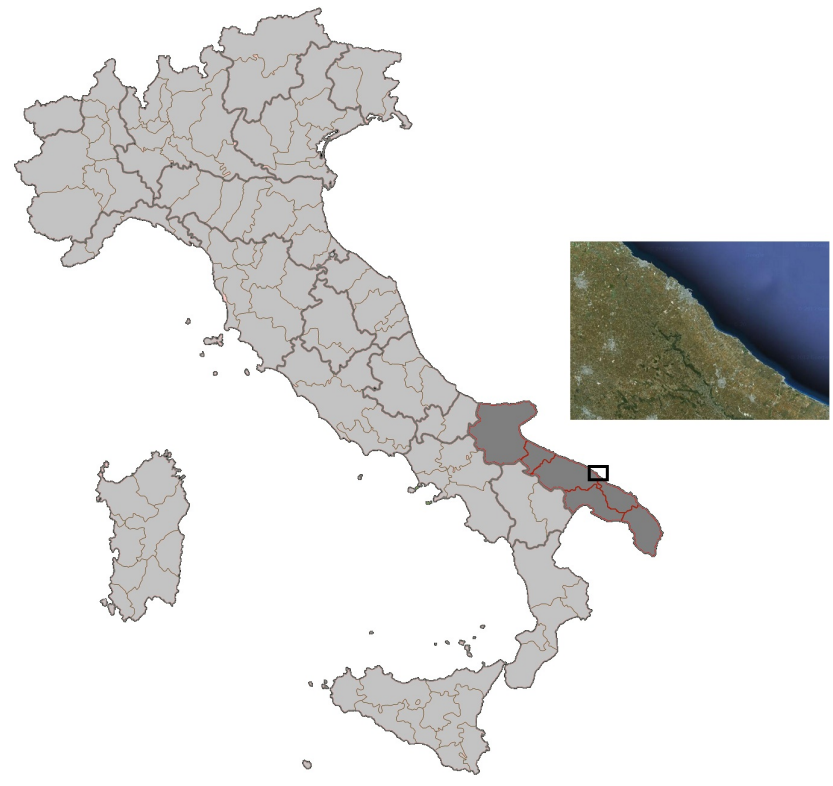

Fig. 1. The localisation of the observed coast in Apulia (the satellite image).

The erosive phenomena are homogeneous for each subambit. Therefore, the measure of erosion, namely criticality, is considered unique for each stripe.

The studied system is based on a continuous assessment of the pressures due to time-changing and space-changing land uses (Di Fazio et al., 2011; Vizzari, 2012); such assessment can be easily integrated with the analysis of criticality and sensitivity provided by RCP for each sub-ambit.

Essential tools to aid the monitoring system are represented by an effective geographic information system (GIS) for consulting and obtaining the necessary data and analysis by the Analytic Hierarchy Process (Cerreta and De Toro, 2010). The acronym, proposed by Saaty (1985) stands for AHP means Analytic (decomposes the problem into its constituent elements) Hierarchy (structure of the constituent elements in a hierarchical manner to the main objective and the sub-goals) Process (processes the data and evaluations in order to achieve the result final).

The evaluation was permitted by satellite land use maps available throughout the region helpful to grouping land-uses in order to characterise concisely the study areas.

By the term criticality, as already said, the greater or lesser propensity to erosion of the coastal area has been indicated; the term sensitivity has been indicated as a level of frailty associated with environmental features and anthropogenic pressures on the context.

The critical erosion of sandy coastline has been classified into high, medium and low. Obviously there was no erosion for calcareous and rocky coast.

The level of criticality was defined according to three indicators: 


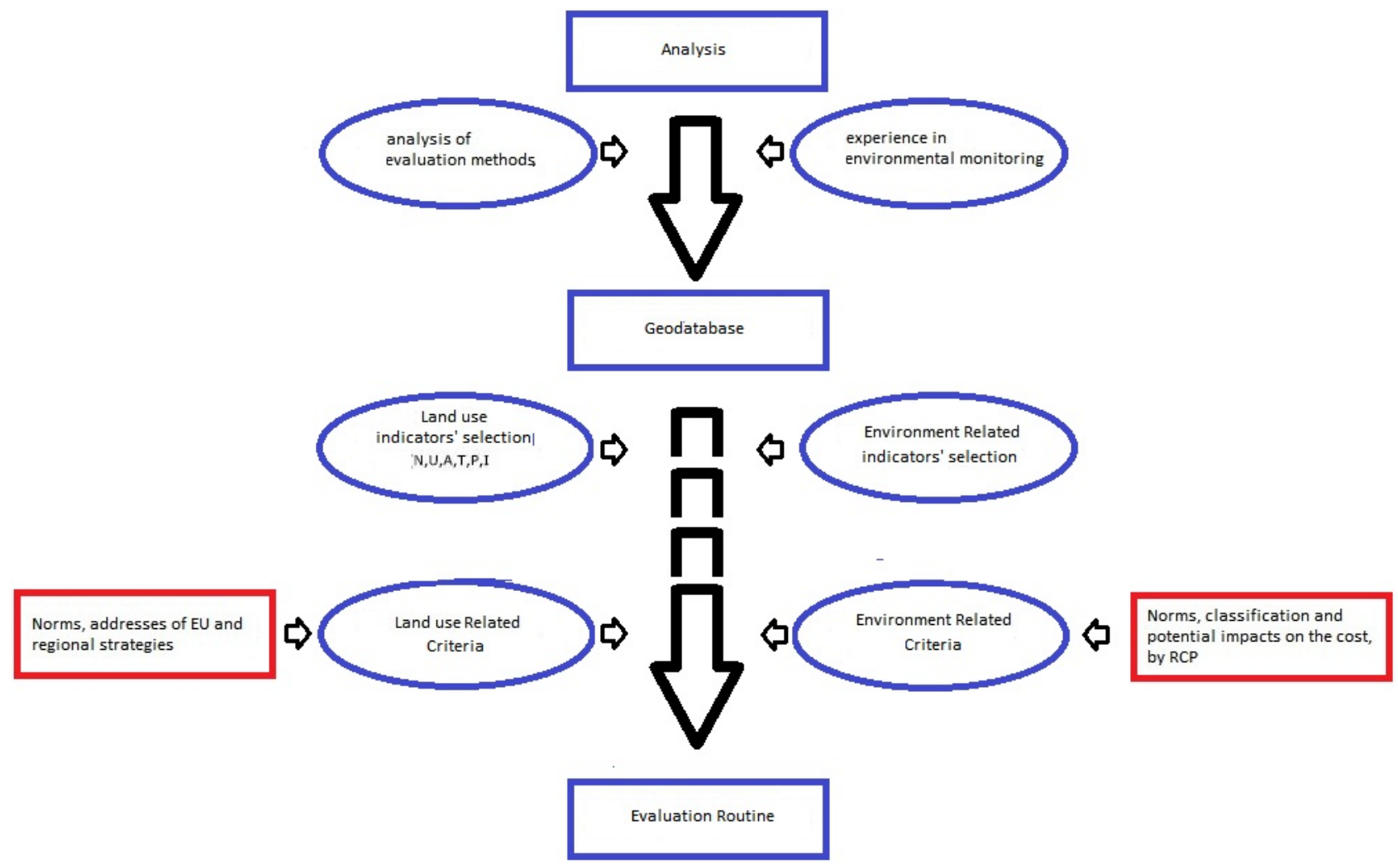

Fig. 2. Logical path of the experiment.

- the historical evolution trend of the coast,

- the evolutionary trend of recently,

- the conservation status of dune systems.

The environmental sensitivity was defined as a complex multi-variable function that represents the physical state of the coast, according to the system of legal protection standards that emphasise the environmental importance.

The sensitivity represents the state of the coastal environment from an historical and an anthropogenic perspective; for this reason a number of criteria have been identified and appropriately weighted, as follows:

- hydrography by a buffer of $300 \mathrm{~m}$ on both sides;

- sites of Community Importance (SCI), Special Protection Areas (SPAs);

- protected Areas and the scope in the Regional Landscape Plan (RLP);

- other extended landscape areas of RLP;

- distinguishable Landscape Areas of RLP:

- the historic settlement patterns;

- use of agricultural land.
The criteria have been "weighted" by the use of AHP.

Using AHP and with the aid of "rating-by-expertise", each hierarchy of land-use criteria has been associated with a weight through the pair wise comparisons.

The criteria were included in a square reciprocal matrix where each row contains the comparison of a given criterion with the other criteria; the comparison is done according to the nine levels of preference (corresponding to the semantic scale of Saaty).

At the end, the software calculates the weights attributed to each of the criteria by constructing a hierarchy between them. After words, each $i$ on $n$ stretch of coast has been provided of a value of Criticaliti $C$ and sensitivity $S$ by

$$
\begin{aligned}
C_{i} & =\sum_{j=1}^{n} \partial_{i j}^{(c)} \gamma_{i j}^{(c)} \\
S_{i} & =\sum_{j=1}^{m} \partial_{i j}^{(s)} \gamma_{i j}^{(s)}
\end{aligned}
$$

where the score of the kroneker numbers $\partial_{i j}^{(s)}$ and $\partial_{i j}^{(c)}$ are assigned according with the Boolean scale:

$$
\begin{aligned}
& \text { presence: } \partial_{i j}^{(s)}=1 \\
& \text { absence : } \partial_{i j}^{(s)}=0
\end{aligned}
$$

the same for $\partial_{i j}^{(c)}$. 
The result of this operation puts each stripe of the coast in a double tree-level classification: high, medium and low environmental criticality/sensitivity.

The different levels of criticality and the erosion of environmental sensitivity were then crossed, giving rise to a classification with nine levels which can provide reference information for the preparation of Municipal Coastal Plan (MCP).

In particular, the classification was as shown in Table 1.

Ultimately, the study has brought a significant contribution to the drafting of appropriate regulatory tools to ensure proper land management and the creation of a knowledge framework that must be continually updated.

For the purposes of the institutional classes of the RCP have the critical task of conditioning the issuance of state concessions, while the classes of environmental sensitivity to influence the types of state concessions and how to contain its impacts.

\section{Dynamic monitoring of values change for coastal areas}

\subsection{General data}

The purpose of this second part of the study was to organise a monitoring system (MS) that can facilitate the control of the changes in the coasts of Apulia: in particular, a support to check and evaluate the real impact of the strategic initiative's plan on the environment and sustainability (Murgante et al., 2010).

The methodology has been structured in relation to the objectives of the monitoring itself, so we opted for structuring an algorithm based on the feedback transmitter capable of communicating with the various phases and operate a continuous cycle.

The basic idea was that the spatial data supported monitoring should be considered a system of alerting, that measuring how fast changes of land use are going on, can bring the attention on measures to adopt for contrasting an excess of carrying capacity of the coastal line.

The land use change can be considered a dummy variable linked with other more complex form of pressure on the environment.

This pressure or causal factor are at the basis of the weighting system.

It was considered appropriate for an assessment of "risk and vulnerability" for the most environmental, such as one arising from the plan, to ensure environmental aspects, but also social and economic. The intersection between the classification of areas interested by the plan and the evaluation of the peculiarities and tendencies of development of the area at the base of the monitoring system so structured, allows a better understanding that facilitates the strategic assessment of the impacts of the initiative.
Table 1. The combination of Criticality and Sensitivity in the Regional Coastal Plan of Apulia.

\begin{tabular}{lllll}
\hline Combination & & Criticality & & Sensitivity \\
\hline C1S1. & C1 & High criticality & S1 & High sensitivity \\
C1S2. & & & S2 & Medium sensitivity; \\
C1S3. & & & S3 & Low sensitivity; \\
\hline C2S1. & C2 & Medium criticality & S1 & High sensitivity; \\
C2S2. & & & S2 & Medium sensitivity \\
C2S3. & & & S3 & Low sensitivity; \\
\hline C3S1. & C3 & Low criticality & S1 & High sensitivity; \\
C3S2. & & & S2 & Medium sensitivity; \\
C3S3. & & & S3 & Low sensitivity \\
\hline
\end{tabular}

Briefly, the algorithm starts from the evaluation of the same aspects such as to characterise the coastal area, as classified by the plan based on the base of criticality and sensitivity. Such information is treated from a socio-economic as well as natural point of view, and constitutes a "system of alerting", relatively to transformations of land in contrast with environmental and landscape peculiarities.

\subsection{The classification of areas of environmental pressure}

To test the system structured as it is taken into account two coastal areas with different characteristics, namely the coastal territory of Monopoli, a medium sized city (about 50000 inhabitants). The inland areas are bordered by a buffer variable that takes into account the physical characteristics of the terrain as defined by the Regional Coastal Plan (RCP).

Since Monopoli comes with a northern rocky coast and in the southern part becomes quite sandy, the areas of study have a substantial variation in the morphology of the coastline.

The coastal line has been divided into three homogeneous areas: a first northern area (named Monopoli 1) is characterised by rocky shoreline and the presence of significant industrial settlements; a second "urban area" is characterised by the harbour infrastructure (Monopoli 2); the third one extends towards south from the end of the municipality (Monopoli 3), characterised by tourist sites of various kinds (holiday homes, villages, residences, beaches and entertainment venues) immersed in an agricultural and natural scenery of some significance given the presence of olive trees.

The logical scheme in the system follows below:

- identifying the scope of study;

- definition of the coastal profile;

- identification of potential impacts within the analysis (through classification of RCP); 
- land uses aggregation on the official regional maps for broad categories $N, U, A, T, P, I$;

- assessment of critical uses well-defined with respect to coastal erosion and environmental sensitivity;

- local and global analysis of variance;

- local analysis of disaggregated indicators.

The first step in the analysis was the choice of indicators for the evaluation of the characters of naturalness, urban relevance, consistency of the port activities, agricultural relevance, importance of tourism, industrial relevance of the area.

To verify the effectiveness of the system, it is then assumed some plausible changes in the area. This change is likely reliable, as they are included as forecast of the City Structure Plan (SP), the interested area, are available in GIS format from the e-planning system.

It is then evaluated the ability of MS to read and grasp their greater or lesser compatibility with the classification of the SP and of the RCP.

As regards RCP, starting from classification based on the criticality and sensitivity, the following aspects are considered essential to characterise a coastal area: naturalness $(N)$, urban land use $(U)$, agricultural land use $(A)$, industrial land use $(I)$, tourist residential land use $(T 1)$ tourist hotel land use $(T)$ harbour areas $(P)$.

The choice of aspects to be monitored was made so that they represent and explain the action plan, simple and easy to interpret, based on readily available data and available, updated and upgraded at regular intervals, capable of showing the trend over time, sensitive and able to advise in relation to trends irreversible, measurable and have a space or georeferenced "footprint".

The source was the classification of land coverage and human land uses deriving from the regional webgis, that refers its classification to Corine Land Cover categories (CLC).

Based on the above shapefile from the land use, there have been other documents created describing aspects $N, U, A$, $T, P$ and $I$, the uses for grouping categories, as below. Categories in CLC are as follows:

- $N$ : coastal lakes and ponds, estuaries, deciduous forests, coniferous forests, areas with sparse vegetation, inland wetlands, mixed coniferous and deciduous forests, meadows and pastures lined with trees, natural pasture, grassland, uncultivated, bushes and shrubs, areas in sclerophyll vegetation, tree-shrub areas evolving; recolonisation areas at artificial surfaces to dense grass cover in proximity of urban green areas,, beaches and sand dunes, bare rocks, cliffs, outcrops, salt marshes, intertidal marine areas, rivers, streams and ditches, canals and waterways, docks without overt productive uses, lagoons. Among these the categories really existing in the area of study are: deciduous forests, coniferous forests, sparse vegetation, mixed coniferous and deciduous forests, meadows and pastures lined with trees, natural pasture, grassland, uncultivated, bushes and shrubs, areas in sclerophyll vegetation; recolonisation areas at artificial surfaces to dense grass cover in the proximity of urban green areas, beaches and sand dunes, bare rocks, outcrops, intertidal marine areas, canals and waterways.

- $U$ : continuous residential fabric, old and dense residential fabric continuous, dense, more recently, low; residential fabric continuous, dense, more recently, high, installation of large systems of public and private hospital settlements, settlements of technological systems; yards, spaces under construction and excavations, sports areas, cemeteries.

- A: productive agricultural settlements; simple arable dry areas; vegetable crops in open fields, greenhouses; simple crops, vegetable crops in open fields, vineyards, olive groves, other permanent crops, temporary crops associated with permanent crops, cropping systems and particle complexes, areas predominantly occupied by agricultural fields with significant areas of natural areas, forestry, soils and reworked artefacts.

- $T 1$ (receptive): campsites, tourist accommodation in bungalows or similar commercial establishment.

- T2 (residential): residential fabric discontinuous residential fabric and rarely nucleiforme; scattered residential fabric.

- $P$ : port areas.

- I: industrial or craft space, outbuildings, abandoned settlements, big plants concentration, networks and areas for distribution, production and transport of energy, mining areas, landfills, junkyards in the open, cemeteries of motor vehicles.

Note that the shapefile land use $T 2$ (residential touristic) was created by grouping all forms of residential fabric discontinuous that in most cases in coastal areas represent holiday homes, in $T$ (receptive) were included all those commercial installations, which as classified in the land uses map as large hotels with attached bathing, as in that coastal line they are clearly prevalent types of settlement.

In more detail, types of land use were ordered by considering the relevance of the extension (local relevance $\gamma$ of land coverage in the context, and on nature) and, as a function of potential negative/positive changes, due to the risk of the variation of urban pressure (respect to the criticality $\omega_{(C)}$ ) and to the increase of erosion (respect to sensitivity $\omega_{(S)}$ ) by a spatial multicriteria approach (Tilio et al., 2012).

To facilitate the operation of pairwise comparison between the issues, three classifications were made to facilitate the 


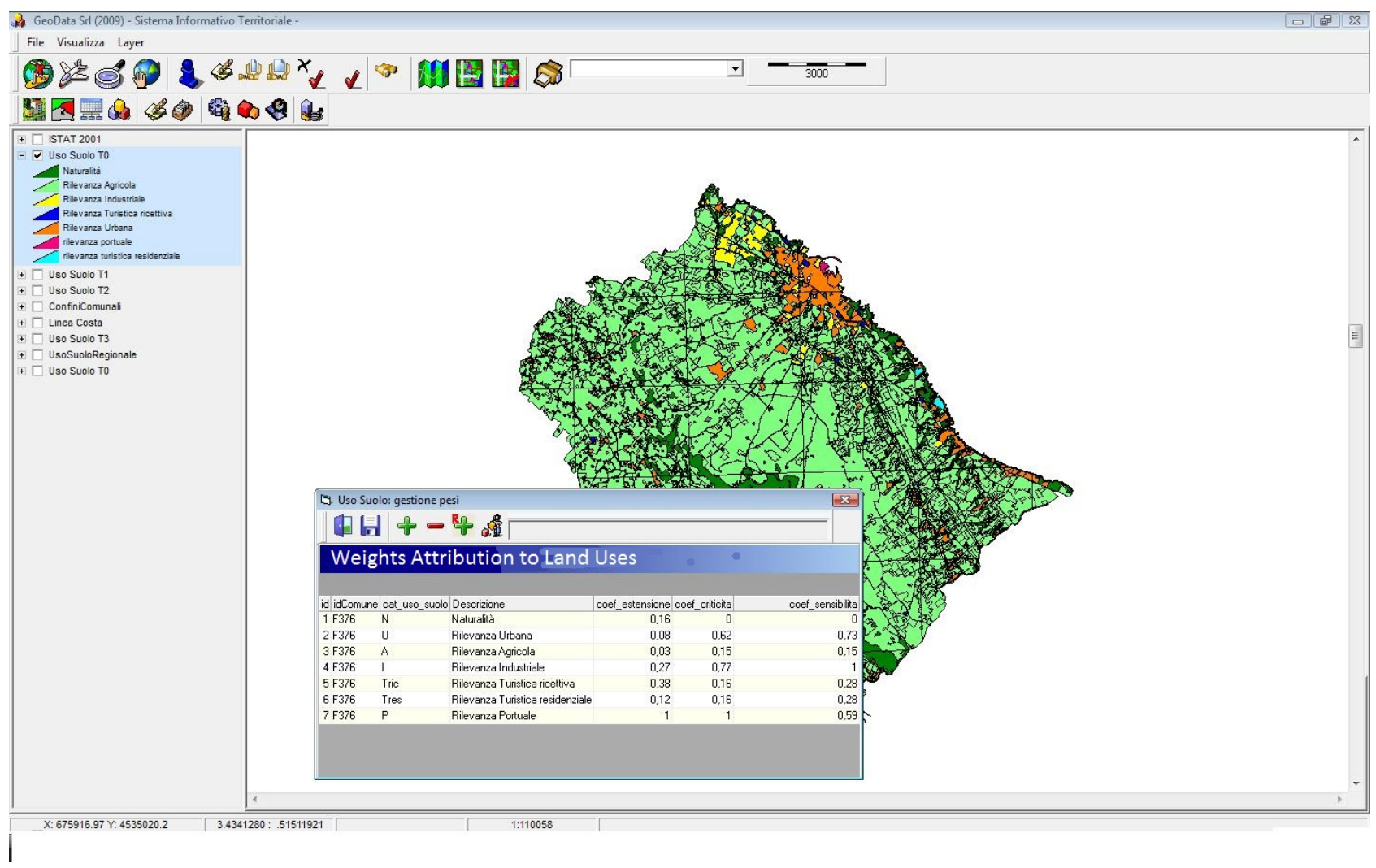

Fig. 3. Weighting according to criticality and sensitivity.

judgments of Saaty's semantic ranking: one, concerning the importance of the extension, relative hazards of the transformation with respect to the critical coastal erosion; another, relative hazards of the transformation with respect to environmental sensitivity (Fusco Girard and De Toro, 2007; Cerreta and Mele, 2012). The result is the weight $(\gamma)$, calculated by the software, as shown in Fig. 3.

The maximum pressure $(100 \%)$ should correspond with the high level of criticality and sensitivity, with the worst category of land use.

After the identification of Saaty's weights, the value was transposed from the typical normalised eigenvalues of Saaty Matrix, to a score 0-1 scale (Table 2).

Therefore, each hectare of industrial land use, located in a PSA weighs the $100 \%$ and each hectare of the naturalised areas weigh the $16 \%$ in terms of environmental pressure.

After weighing the relevance on pressure of land uses, this relevance should be crossed with the average level of pressure on sensitivity and criticality in each PSA.

Given the category of land use in the census section $\mathrm{CS}_{(X)}$,
Table 2. Coefficient of extension $\gamma$.

\begin{tabular}{lccccccc}
\hline Land use & $N$ & $U$ & $A$ & $T 1$ & $T 2$ & $P$ & $I$ \\
\hline Extension $\gamma$ & 0.16 & 0.08 & 0.03 & 0.38 & 0.12 & 1.00 & 0.27 \\
\hline
\end{tabular}

Given the category of land use $X$, given the seven criteria

$$
\begin{aligned}
& P_{(X)}=f\left(\Sigma_{i} \alpha_{i} \gamma_{i} \omega_{C i}, \quad \Sigma_{i} \beta_{i} \gamma_{i} \omega_{S i}\right) \\
& I=1 \text { to } 6 \\
& \omega_{C}=1.00=>\text { criticality }=C 1 \\
& \omega_{C}=0.66=>\text { criticality }=C 2 \\
& \omega_{C}=0.33=>\text { criticality }=C 3 \\
& \omega_{S}=1.00=>\text { sensitivity }=S 1 \\
& \omega_{S}=0.66=>\text { sensitivity }=S 2 \\
& \omega_{S}=0.33=>\text { sensitivity }=S 3 .
\end{aligned}
$$

It has been possible to obtain the matrices of the Saaty pairwise comparisons and to determine all land use coefficients $\alpha_{N}, \alpha_{U}, \alpha_{A}, a_{I}, \alpha_{T}, \alpha_{P}$, and $\beta_{N}, \beta_{U}, \beta_{A}, \beta_{I}, \beta_{T}, \beta_{P}$, that respectively measure the general contribution (in the way $\omega$ represents the local contribution) for criticality and sensitivity. Table 3 shows the weighted pressure for each area (namely the values of $\Sigma_{i} \alpha_{i} \gamma_{i} \omega_{C i}$ and $\Sigma_{i} \beta_{i} \gamma_{i} \omega_{S i}$ ) (Table 4). 


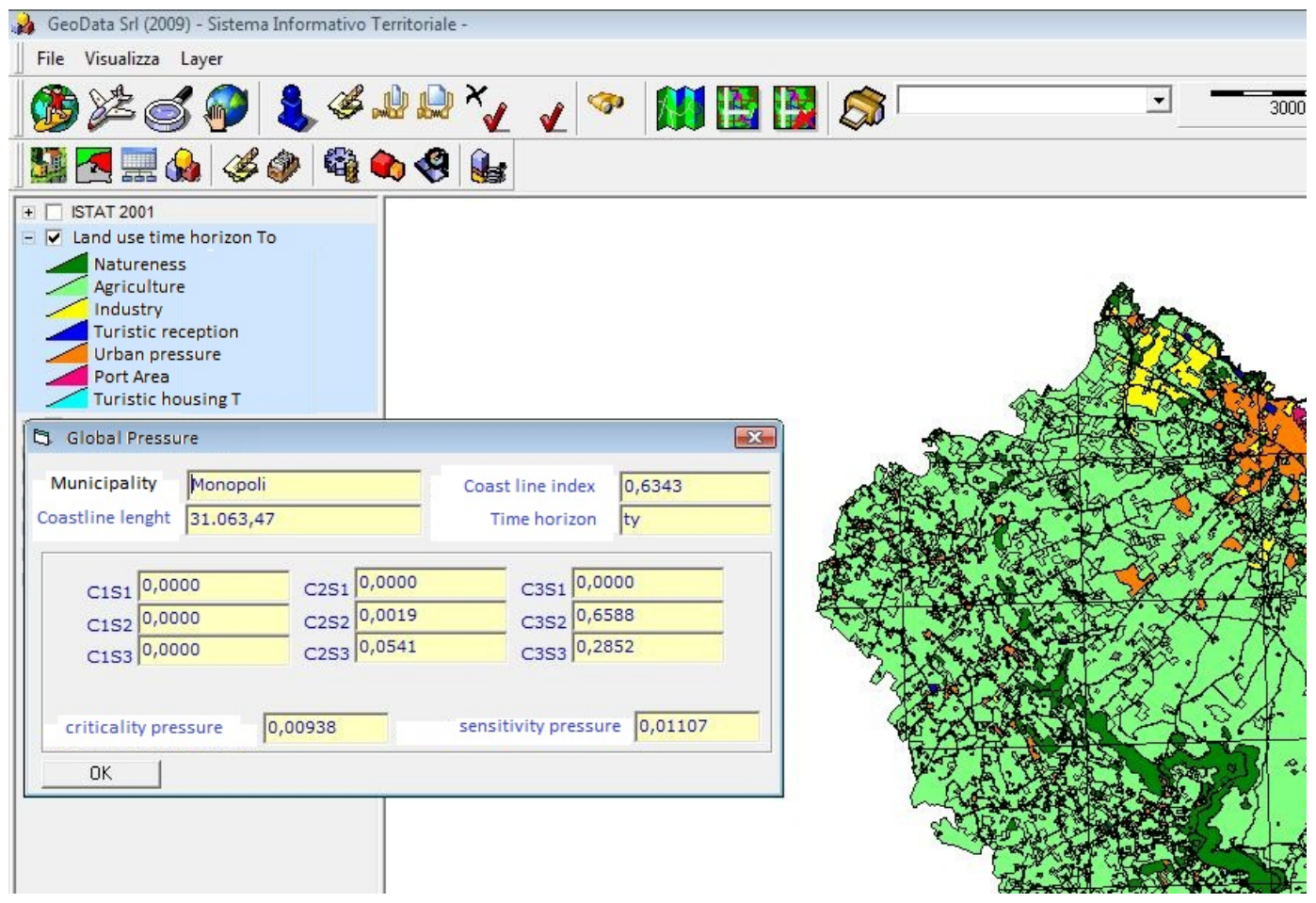

Fig. 4. Calculation at time $T_{y}$ the change of global pressures due to criticality and sensitivity according to Weighted Sum of Land Use Pressures $(N, T, I, U, A, P)$.

Figure 4 shows the calculation on the software of the components of pressure $\left(C_{i} S_{j}\right.$, where $i=1$ to 3 and $j=1$ to $3-$ weak, medium and strong level of Criticality and Sensitivity).

\subsection{Profiling the issues of coastal municipalities}

Based on this first trial, as part of a research project funded by the Region Apulia, in collaboration with Polytechnic of Bari and the company Geodata S.R.L., we proceeded to the realisation of a software (identified by the acronym MOCA: Monitoring Of Coastal Areas). MOCA is able to integrate the evaluation routine concerned with GIS technologies and an alerting system, in order to profile differently each coastal municipality of the Region. The software is designed in order to manage a spatial data infrastructure (SDA), which will facilitate the reading of the ongoing and potential changes, arising from a comparison between what are the provision of plans, programmes or interventions, the SEA of RCP, and the analysis of the real land use changes (Prezioso, 2003), and the consequent effect of changes on RCP criticality and sensitivity.

The idea is that the MOCA System could measure the ratio among land use changes, measured by the variation of a synthetic index and a given time interval, that can be coherent with the ordinary upgrade of a Plan. The time length, for instance, could be a period of five years, in the case of the study, that is the frequency of upgrading the operative programme of the General Urban Plan in Apulia.
Table 3. Coefficients of criticality $\alpha$ and sensitivity $\beta$.

\begin{tabular}{lcccccc}
\hline Land use & $N$ & $U$ & $A$ & $T 1+T 2$ & $P$ & $I$ \\
\hline criticality $\alpha$ & 0.06 & 0.62 & 0.15 & 0.26 & 1.00 & 0.77 \\
sensitivity $\beta$ & 0.06 & 0.73 & 0.15 & 0.28 & 0.59 & 1 \\
\hline
\end{tabular}

The software can potentially work on a larger SDA: in fact, spatial data relating to land use (aggregate indicators) are combined and joined together with other various data, useful to investigate situations of risk and danger, coming from different sources (local GIS, web GIS, data from the national institute of statistics and so on).

The scales of analysis allowed by the software are variable; the validation of the software was done working on a municipal scale, using the assessment of land use areas defined by administrative features.

The Municipality was subdivided into subareas, coincident with sections (CS) identified in the subdivision of territory provided by national population census. For each subarea, the same indicators were calculated from the first case: the pressure given by $N, U, A, T, P, I$, weighted for sensitivity and criticality.

A choice of this kind, however, involves the risk of evaluating in the same manner similar transformations in the common characterised by a different "coastal character". This risk is due to the need to manage differently the same land 
Table 4. Adjusted pressure areas according to weighted coefficient of criticality and sensitivity for monitoring the change due to City plan implementation.

\begin{tabular}{|c|c|c|c|c|c|c|c|}
\hline Area & & $\begin{array}{c}N \\
\left(\alpha_{N}, \beta_{N}\right)\end{array}$ & $\begin{array}{c}U \\
\left(\alpha_{U}, \beta_{U}\right)\end{array}$ & $\begin{array}{c}A \\
\left(\alpha_{A}, \beta_{A}\right)\end{array}$ & $\begin{array}{c}I \\
\left(\alpha_{I}, \beta_{I}\right)\end{array}$ & $\begin{array}{c}T 1+T 2 \\
\left(\alpha_{T}, \beta_{T}\right)\end{array}$ & $\begin{array}{c}P \\
\left(\alpha_{P}, \beta_{P}\right)\end{array}$ \\
\hline Weights & & $\gamma_{N}$ & $\gamma_{U}$ & $\gamma_{A}$ & $\gamma_{I}$ & $\gamma_{T}$ & $\gamma_{P}$ \\
\hline Monopoli1 $\left(c_{x}, s_{y}\right)$ & $(3.0,2.6)$ & 1.4800 & 0.3488 & 2.1258 & 0.5968 & 0.0000 & 3.5613 \\
\hline $\operatorname{Monopoli2}\left(c_{x}, s_{y}\right)$ & $(3.0,2.6)$ & 1.8705 & 2.5808 & 1.5036 & 0.5538 & 1.7000 & 0.6102 \\
\hline $\operatorname{Monopoli3}\left(c_{x}, s_{y}\right)$ & $(2.8,2.2)$ & 2.1152 & 0.1232 & 2.3502 & 1.4894 & 0.0000 & 0.0084 \\
\hline
\end{tabular}

use category in several contexts: the land use can have different pressure levels for each different municipality.

To remedy the highlighted problems, the following steps were taken in the testing phase to implementing data capable of profiling in a simple and accessible system the "coastrelated" issues of each joint territory.

The means for this characterisation is represented by a set of indicators, which are available and will be available for all common with part of the "wet" perimeter, these are:

a. length of the coastline town;

b. the ration between length of coast line city on municipal boundary, multiplied for two;

c. length of areas classified by potential effects in RCP/ length of coast line city.

These indicators, suitably used in the routine of evaluation, help to refer the changes to the environment and the coastal issues, "profiling" the territories.

The maximum pressure will correspond:

a. to the value 1 where the territory is completely urbanised,

b. to an absolutely linear shape (and the perimeter is composed by two parallel lines on the coast), and

c. to the amount of potential environmental effects to investigate so that the RCP identifies each area according to its level of criticality and sensitivity,

Figure 4 also shows the computation of the coastal "shape coefficient" in the software, that is a function of the three above-mentioned indicators.

The software MOCA, from both theoretical and practical gathered information, allows a uniform assessment of the environmental pressure caused by different land uses, with particular reference to critical coastal erosion and environmental sensitivity. The assessment may be conducted within the selected study, this according to some simple indicators is "profiled". The analyses are, thus, related fields of study so as to be comparable between different areas. The assessment of the land use is first an information layer, following this localised analysis of disaggregated indicators collected in databases that can be implemented continuously.
A significant aspect is related to adaptability to local contexts and coastal profiles of different sizes for analysis in different contexts and physical characteristics of size.

The possibility of identifying a field of study and the association of simple indicators for its characterisation allows to opt for areas defined by administrative boundaries (as in the case of experimentation), but also through character definitions of physic-morphological, sometimes more suited to analysis. In fact, it becomes possible to manage each municipality that owns a coast line, by considering in the same time natural constrains, land use constrains, and relevance to the physiographic coastal unit of the entire territory (the complete evaluation logframe is shown in Fig. 5).

\section{Perspectives and remarks}

The introduction of the "shape coefficient" allows, beyond the definition of the type of choice, of "weigh" the coastal character on the whole municipal area. This weighing system allows to compare different municipalities and permits to assume a common alerting threshold, as the primary problem is the definition of a non-value. The indicators chosen for profiling are valid for coastal areas of variable geometry and extension; therefore, the possibility to perform the analyses at any scale, relative to the needs identified, is allowed.

The association to each area of a database consents to profile the areas of major interest, since they are subject to change or because exerting environmental pressure increased, more detailed analyses by dynamically monitored indicators.

The indicators covered by this analysis may also vary depending on the needs, because the databases are continuously updated and implemented. The cognitive maps produced by the software provides an excellent overview of state and forecasts.

The same theoretical and methodological steps taken to build the product are still replicable to other assessments, keeping the basic knowledge fixed on the classification of land uses (Cerreta and De Toro, 2012; Fichera et al., 2011, 2012).

However, it is not possible, without a real experimentation in other fields, to assess whether the routine, structured as follows, although replicable, is the most appropriate for subjects 
Assessment of land use pressure

$$
\text { respect to RCP norms: }
$$

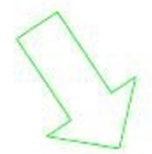

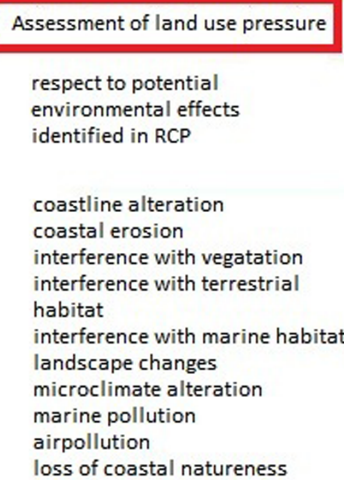

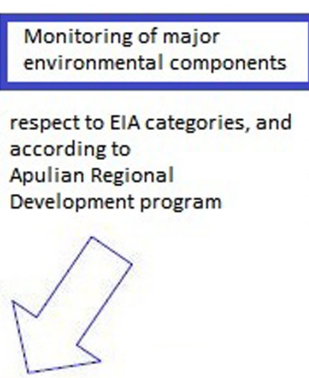
Air Water Soil Ecosystems Marine Habitat Energy Waste transport Public Healt

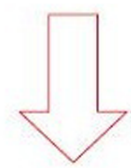

Fig. 5. The integrated process of evaluation.

of a different nature. Either way, the product offers the possibility, through a simple user interface and at the same time flexible, to restructure the coefficients of impact in relation to different issues and to implement cognitive-different regulatory frameworks. It seems clear, however, that only a professional, experienced in assessment methodologies, can consistently achieve a multi-criteria evaluation routine that can be imported into the system.

The evaluation system, fully implemented in software design, is sensitive to change in territory and allows an assessment with regard to global and local land use more or less compatible with coastal issues. It also allows you to render the results of analyses using maps and cognitive evaluation.

Important results have shown the ability to monitor, in addition to land use and classification of RCP, any activity through appropriately chosen indicators, according to local situations (the trial included a national statistic database, but nothing prevents you to widen or narrow the field of analysis as needed), the possibility of covering the entire region by comparing the analysis to settings with different coastal characteristics; the chance to work on different spatial scales, and finally the possibility to adapt the software to other developments in evaluations of different genres.

Adaptability, flexibility, uniformity of analysis are the characteristics sought in the realisation of the product and, as tested, meets these requirements.

Acknowledgements. The authors thank their colleagues Giovanni La Trofa and Pasquale Balena for continuing support in developing the software and for their profitable suggestion during many interesting discussions.
Edited by: B. Murgante

\section{References}

Arts, J.: EIA Follow-Up: On the Role of Ex-post Evaluation in Environmental Impact Assessment, Geo Press, Groningen, 1998

Bencardino, M.: A Spatial Decision Support System for the Strategic Environmental Assessment of General Town Planning of Casalbore district (Av), in: Proceedings of the Fifth European Congress on Regional Geoscientific Cartography and Information Systems (ECONGEO 2006), 13-16 June 2006, Volume I, Barcelona, Spain, 437-439, 2006.

Cerreta, M. and De Toro, P.: Integrated spatial assessment for a creative decision-making process: A combined methodological approach to strategic environmental assessment, Int. J. Sustain. Dev., 13, 17-30, 2010.

Cerreta, M. and De Toro, P.: Assessing urban transformations: A SDSS for the master plan of Castel Capuano, Naples", Lect. Notes Comput. Sci., 7334, 168-180, 2012.

Cerreta, M. and Mele, R.: A landscape complex value map: integration among soft values and hard values in a spatial decision support, Lect. Notes Comput. Sci., 7334, 653-659, 2012.

Cerreta, M., Panaro, S., and Cannatella, D.: Multidimensional spatial decision-making process: Local shared values in action, Lect. Notes Comput. Sci., 7334, 54-70, 2012.

Di Fazio, S., Modica, G., and Zoccali, P.: Evolution trends of land use/land cover in a Mediterranean forest landscape in Italy, Lect. Notes Comput. Sci., 6782, 284-299, 2011.

Fichera, C. R., Modica, G., and Pollino, M.: GIS and remote sensing to study urban-rural transformation during a fifty-year period, Lect. Notes Comput. Sci., 6782, 237-252, 2011.

Fichera, C. R., Modica, G., and Pollino, M.: Land Cover classification and change-detection analysis using multi-temporal remote sensed imagery and landscape metrics, Ital. J. Remote Sens., 45, 1-18, 2012. 
Fischer, T. B.: Theory and Practice of Strategic Environmental Assessment, Earthscan, London, 2007.

Fischer, T. B. and Gazzola, P.: SEA effectiveness criteria - equally valid in all countries? The case of Italy, Environ. Impact Assess. Rev., 26, 396-409, 2006.

Fusco Girard, L. and De Toro, P.: Integrated spatial assessment: A multicriteria approach to sustainable development of cultural and environmental heritage in San Marco dei Cavoti, Italy, Centr. Eur. J. Operat. Res., 15, 281-299, 2007.

Kornov, L. and Thissen, W.: Rationality in Decision and PolicyMaking: Implications for Strategic Environmental Assessment, Impact Assess. Project Appr., 18, 191-200, 2000.

Marshall, R: Environmental impact assessment follow-up and its benefits for industry, Impact Assess. Project Appr., 23, 191-196, 2005.

McCallum, D: Planned follow-up, a basis for acting on EIAs, Proceedings of the Annual Meeting of the International Association for Impact Assessment, Utrecht, 27-28 June 1985.

Morrison-Saunders, A., Marshall, R., and Arts, J.: Eia Follow up: International Best Practice Principles, in: Special Publication Series No. 6, International Association for Impact Assessment, Fargo, USA, 2007.

Murgante, B., Borruso, G., and Lapucci, A.: Sustainable Development: concepts and methods for its application in urban and environmental planning, in: Geocomputation, Sustainability and Environmental Planning, Studies in Computational Intelligence, edited by: Murgante, B., Borruso, G., and Lapucci, A., SpringerVerlag, Berlin, 348, 1-15, 2010.
Partidário, M. R.: Elements of an SEA framework - improving the added-value of SEA, Environ. Impact Assess. Rev., 20/6, 647663, 2000.

Partidario, M. R. and Arts, J.: Exploring the Concept of Strategic Environmental Assessment Follow-Up, Impact Assess. Project Appr., 23/3, 246-257, 2005.

Prezioso, M.: Pianificare in sostenibilità, Natura e finalità di una nuova politica per il governo del territorio, AdnkronosLibri, Roma, 2003.

Saaty, T.: The Analytic Hierarchy Process: Planning, Priority Setting, Resource Allocation, McGraw-Hill, New York, 1985.

Sadler, B. and Verheem, R.: Strategic Environmental Assessment Status, Challenges and Future Directions, Ministry of Housing, Spatial Planning and the Environment, The Hague, 1996.

Selicato, M., Torre, C. M., and La Trofa, G.: Prospect of Integrate Monitoring: A Multidimensional Approach, Lect. Notes Comput. Sci., 7334, 144-156, 2012.

Sheate, W. R.: Tools, techniques \& approaches for sustainability, World Scientific Publishing, Singapore, 2010.

Tilio, L., Murgante, B., Di Trani, F., Vona, M., and Masi, A.: Mitigation of urban vulnerability through a spatial multicriteria approach, Disaster Adv., 5, 138-143, 2012.

Vizzari, M.: Spatio-temporal analysis using urban-rural gradient modelling and landscape metrics, Lect. Notes Comput. Sci., 6782, 103-118, 2012. 